\title{
Molecular imaging and fluid biomarkers of Alzheimer's disease neuropathology: an opportunity for integrated diagnostics
}

\author{
Valentina Garibotto ${ }^{1,2}$ (D) Marina Boccardi ${ }^{3} \cdot$ Arturo Chiti $^{4,5} \cdot$ Giovanni B. Frisoni ${ }^{6,7}$ \\ Published online: 10 March 2021 \\ (C) Springer-Verlag GmbH Germany, part of Springer Nature 2020
}

\begin{abstract}
Alzheimer's disease (AD) gold standard diagnosis is the detection of amyloid plaques and neurofibrillary aggregates of hyperphosphorylated tau at autopsy. The developments in the diagnostic field over the last two decades have made these neuropathological processes partly accessible in vivo by two main classes of biomarkers: molecular imaging and laboratory measures. Molecular imaging, using specific PET radiotracers, allows visualizing the presence of amyloid and tau in the brain; laboratory assessments provide indirect evidence measuring the release or retention of specific amyloid and tau isoforms in the cerebrospinal fluid (CSF) and more recently, with the development of high-sensitivity systems, also in plasma.

Over the past few years, there have been major advances both in the molecular imaging and fluid biomarker research. In order to be employed in clinical practice, biomarkers should be investigated systematically for their validity, i.e., their ability to discriminate true-positive from true-negative cases accurately and consistently across clinical contexts. The main
\end{abstract}

This article is part of the Topical Collection on Neurology - Dementia

Valentina Garibotto

valentina.garibotto@hcuge.ch

$1 \quad$ NIMTlab - Neuroimaging and Innovative Molecular Tracers Laboratory, University of Geneva, Geneva, Switzerland

2 Nuclear Medicine and Molecular Division, University Hospitals of Geneva, Rue Gabrielle-Perret-Gentil 4, 1205 Geneva, Switzerland

3 German Center for Neurodegenerative Diseases (DZNE) Rostock-Greifswald, Rostock, Germany

4 Department of Biomedical Sciences, Humanitas University, Milan, Italy

5 Humanitas Clinical and Research Center, IRCCS, Milan, Italy

6 Memory Clinic, University Hospital, Geneva, Switzerland

7 LANVIE - Laboratory of Neuroimaging of Aging, University of Geneva, Geneva, Switzerland steps of the validation process of AD biomarkers have been formalized in a Strategic Biomarker Roadmap, resulting from the collaborative work of a group of experts in the diagnosis of cognitive disturbances and biomarkers [1]. The roadmap, updated based on recent theoretical advancement [2], outlines the methodology required to produce and assess evidence of analytical validity, clinical validity, and clinical utility for each biomarker. This framework provides therefore a systematic tool to identify the gaps in knowledge that should be filled with priority to allow evidence-based clinical and policy decisions. Only once the analytical and clinical validity of a biomarker are proven can its utility (i.e., the impact of the biomarker-based diagnosis on clinically relevant outcomes) be properly assessed.

PET tracers specific for amyloid pathology, tested in clinical studies since 2004, have already proven their analytical validity and are currently evaluated in large-scale phase 4 studies testing clinical validity and collecting data for clinical utility $[3,4]$. Tracers specific for tau pathology represent a more recent advance. The first ligands described, the so-called first-generation tracers, have been introduced in clinical studies in 2013. Among these, 18F-flortaucipir (Tauvid) was approved for use by the Food and Drug Administration earlier this year (https:// www.fda.gov/drugs/drug-approvals-and-databases/drugtrial-snapshot-tauvid). In parallel, a number of new ligands (second-generation tracers), purportedly with greater sensitivity and specificity, are currently under evaluation. Their level of maturity within the Strategic Biomarker Roadmap structure is summarized in the contributions by Wolters, Chiotis, and Bischof [5-7].

Laboratory assessments of amyloid and tau pathology have also significantly advanced over the last years. CSF measures, currently routinely used in clinical practice, have improved their standardization across centers through unified protocols for sampling, handling, and storage and the introduction of fully automated assays [8]. The introduction of novel technologies with greatly increased sensitivity has also allowed the 
reliable detection of the molecular isoforms of amyloid and tau in the plasma, showing excellent accuracy to discriminate true positives from true negatives [9].

These considerable advances in molecular imaging and fluid biomarkers emphasize the need to direct focused efforts to their clinical translation. One of the crucial steps in this direction is their comparison and combination, as highlighted by the roadmap structure. Comparative data between novel and established diagnostic procedures are required to establish evidence-based standards of best care. This entails finding strategies to quantify and compare complex dimensions that, beyond analytical and clinical validity, include costs, accessibility, and patient preferences and impact on health care and society. Multiple studies have shown that the results of molecular imaging and fluid assessment of the same molecular target, amyloid or tau, are correlated, but the agreement among measures is not perfect and variable across studies $[8,9]$. This is linked to the intrinsic nature of the test: molecular imaging tools can follow pathology accumulation across different brain topography stages, while changes in fluid might be more sensitive to the earliest change of state, from negative to positive, less sensitive to disease progression over more advanced stages and of course insensitive to topography. These diagnostic tools differ not only in terms of accuracy across disease phases but also in terms of accessibility, invasiveness, and costs. Comparative studies should namely measure the incremental diagnostic value provided by a test, i.e., not only its diagnostic performance but also its added value when other information, for example the result of gateway or other tests, is already available. There are only a few diagnostic studies measuring this parameter [10-12], one in this issue comparing the incremental diagnostic value of amyloid PET and tau PET [13]. The limited evidence on the comparison and combination of biomarkers presumably contributes to explain the variable policies for coverage across countries and the lack of widely accepted detailed diagnostic algorithms $[1,2]$. Moreover, diagnostic algorithms need to be specific for different contexts of use. The recently developed opportunity to measure pathology in plasma, in particular, opens an unprecedented perspective of non-invasive and low-cost assessment, which could be proposed as the gateway to more invasive and expensive tests, such as CSF and imaging, within the usual diagnostic procedure, and may also be used for population screening. For the first scenario, some studies already propose adapted thresholds in view of subsequent investigations of amyloid status by amyloid PET, although not yet for tau $[14,15]$.

One of the obstacles to build the evidence needed to compare and combine biomarkers might lag in the fact that biomarkers in $\mathrm{AD}$ stem from two different areas of diagnostic medicine, namely imaging and laboratory medicine, most often working as independent entities with limited exchanges. These disciplines are split in terms of training, technological platforms, and clinical reporting and workflow, despite sharing questions, objectives, and often challenges. The larger introduction of information technology cannot per se overcome this dichotomy, as diagnostic decision support systems, which ideally would be able to combine data of different nature, are most commonly designed to guide only the choice for the best imaging test or the best laboratory test to be recommended in a given condition [16]. An increasingly advocated approach, already a reality in some institutions, is to enhance the cross-talk between diagnostic disciplines into an "integrated diagnostics" approach [17, 18]. Multiple studies have shown an added value of the integration of results of different modalities, e.g., imaging and pathology or imaging and laboratory tests, in oncology, cardiovascular diseases, and infectious diseases [19]. The combination of different tests in integrated reports and multidisciplinary analyses has been shown to reduce the rate of misdiagnoses and equivocal findings in these clinical fields and improve management in case of conflicting results [18].

In our view, the implementation of "integrated diagnostics" in $\mathrm{AD}$ could be beneficial in many respects. Integrated reports of imaging and fluid findings could leverage on the strengths and weaknesses of both techniques with an added diagnostic value. The collection of comparative evidence could be facilitated by an increased collaboration across fields. Finally, experts in integrated diagnostics in $\mathrm{AD}$ would be the ideal partner for clinicians to appropriately build diagnostic algorithms and accelerate an evidence-based larger scale adoption of AD biomarkers.

\section{Compliance with ethical standards}

Conflict of interest Valentina Garibotto reports grants from the Swiss National Science Foundation (projects 320030_169876, 320030_185028, and IZSEZ0_188355) and the Velux Foundation (project 1123). Garibotto received financial support for research and/or speaker fees through her institution from Siemens Healthineers, GE Healthcare, Life Molecular Imaging, Cerveau Technologies, Roche, and Merck. Giovanni B. Frisoni reports grants from Alzheimer Forum Suisse, Académie Suisse des Sciences Médicales, Avid Radiopharmaceuticals, Biogen, GE International, Guerbert, Association Suisse pour la Recherche sur l'Alzheimer, IXICO, Merz Pharma, Nestlé, Novartis, Piramal, Roche, Siemens, Teva Pharmaceutical Industries, Vifor Pharma, and Alzheimer's Association; he has received personal fees from AstraZeneca, Avid Radiopharmaceuticals, Elan Pharmaceuticals, GE International, Lundbeck, Pfizer, and TauRx Therapeutics.

Arturo Chiti received speaker honoraria from General Electric and Blue Earth Diagnostics, acted as a scientific advisor for Blue Earth Diagnostics and Advanced Accelerator Applications, and benefited from an unconditional grant from Sanofi to Institution.

Boccardi has no conflicts of interest to disclose.

Ethical approval Institutional Review Board approval was not required because the paper is an Editorial.

Informed consent Not applicable. 


\section{References}

1. Frisoni GB, Boccardi M, Barkhof F, Blennow K, Cappa S, Chiotis $\mathrm{K}$, et al. Strategic roadmap for an early diagnosis of Alzheimer's disease based on biomarkers. Lancet Neurol. 2017;16:661-76. https://doi.org/10.1016/S1474-4422(17)30159-X.

2. Boccardi M, Dodich A, Albanese E, Gayet-Ageron A, Festari C, Walter MA, et al. The Strategic Biomarker Roadmap for the validation of Alzheimer's diagnostic biomarkers: methodological update. Eur J Nucl Med Mol Imaging. https://doi.org/10.1007/ s00259-020-05120-2

3. Frisoni GB, Barkhof F, Altomare D, Berkhof J, Boccardi M, Canzoneri E, et al. AMYPAD diagnostic and patient management study: rationale and design. Alzheimers Dement. 2019;15:388-99. https://doi.org/10.1016/j.jalz.2018.09.003.

4. Rabinovici GD, Gatsonis C, Apgar C, Chaudhary K, Gareen I, Hanna L, et al. Association of Amyloid Positron Emission Tomography with subsequent change in clinical management among Medicare beneficiaries with mild cognitive impairment or dementia. JAMA. 2019;321:1286-94. https://doi.org/10.1001/ jama.2019.2000.

5. Wolters E, Dodich A, Boccardi M, Corre J, Drzezga A, Hansson O, et al. Clinical validity of increased cortical uptake of $[18 \mathrm{~F}]$ flortaucipir on PET as a biomarker for Alzheimer's disease in the context of a structured 5-phase biomarker development framework. Eur J Nucl Med Mol Imaging. https://doi.org/10. 1007/s00259-020-05118-w

6. Chiotis K, Dodich A, Boccardi M, Festari C, Frisoni GB, Garibotto $\mathrm{V}$, et al. Clinical validity of increased cortical uptake of tau ligands of the THK family and 11C-PBB3 on PET as biomarkers for Alzheimer's disease in the context of a structured 5-phase development framework. Eur J Nucl Med Mol Imaging. https://doi.org/10. 1007/s00259-021-05277-4

7. Bischof GN, Dodich A, Boccardi M, van Eimeren T, Festari C, Barthel $\mathrm{H}$, et al. Clinical validity of increased cortical uptake of second-generation Tau PET tracers as a biomarker for Alzheimer's disease in the context of a structured 5-phase development framework. Eur J Nucl Med Mol Imaging. https://doi.org/10. 1007/s00259-020-05156-4

8. Leuzy A, Ashton NJ, Mattsson-Carlgren N, Dodich A, Boccardi M, Corre J, et al. Update on the clinical validity of cerebrospinal fluid amyloid, tau, and phospho-tau as biomarkers for Alzheimer's disease in the context of a structured 5-phase development framework. Eur J Nucl Med Mol Imaging. 2020. https://doi.org/10.1007/ s00259-021-05258-7

9. Ashton NJ, Leuzy A, Karikari TK, Mattsson-Carlgren N, Dodich A, Boccardi M, et al. Validation status of blood biomarkers of amyloid and phospho-tau assessed with the 5-phase development framework for AD biomarkers. Eur J Nucl Med Mol Imaging. https://doi.org/10.1007/s00259-021-05253-y

10. Massa F, Farotti L, Eusebi P, Capello E, Dottorini ME, Tranfaglia $\mathrm{C}$, et al. Reciprocal incremental value of $18 \mathrm{~F}-\mathrm{FDG}-\mathrm{PET}$ and cerebrospinal fluid biomarkers in mild cognitive impairment patients suspected for Alzheimer's disease and inconclusive first biomarker. J Alzheimers Dis. 2019;72:1193-207. https://doi.org/10.3233/ JAD-190539.

11. Perini G, Rodriguez-Vieitez E, Kadir A, Sala A, Savitcheva I, Nordberg A. Clinical impact of (18)F-FDG-PET among memory clinic patients with uncertain diagnosis. Eur J Nucl Med Mol Imaging. 2020. https://doi.org/10.1007/s00259-020-04969-7.

12. Ramusino MC, Garibotto V, Bacchin R, Altomare D, Dodich A, Assal F, et al. Incremental value of amyloid-PET versus CSF in the diagnosis of Alzheimer's disease. Eur J Nucl Med Mol Imaging. 2020;47:270-80. https://doi.org/10.1007/s00259-019-04466-6.

13. Altomare D, Caprioglio C, Assal F, Allali G, Mendes A, Ribaldi F, et al. Diagnostic value of amyloid-PET and tau-PET: a head-tohead comparison. Eur J Nucl Med Mol Imaging. https://doi.org/ 10.1007/s00259-021-05246-x

14. Verberk IMW, Slot RE, Verfaillie SCJ, Heijst H, Prins ND, van Berckel BNM, et al. Plasma amyloid as prescreener for the earliest Alzheimer pathological changes. Ann Neurol. 2018;84:648-58. https://doi.org/10.1002/ana.25334.

15. Palmqvist S, Janelidze S, Stomrud E, Zetterberg H, Karl J, Zink K, et al. Performance of fully automated plasma assays as screening tests for Alzheimer disease-related beta-amyloid status. JAMA Neurol. 2019. https://doi.org/10.1001/jamaneurol.2019.1632.

16. Sutton RT, Pincock D, Baumgart DC, Sadowski DC, Fedorak RN, Kroeker KI. An overview of clinical decision support systems: benefits, risks, and strategies for success. NPJ Digit Med. 2020;3:17. https://doi.org/10.1038/s41746-020-0221-y.

17. Krestin GP, Grenier PA, Hricak H, Jackson VP, Khong PL, Miller $\mathrm{JC}$, et al. Integrated diagnostics: proceedings from the 9th biennial symposium of the International Society for Strategic Studies in radiology. Eur Radiol. 2012;22:2283-94. https://doi.org/10.1007/ s00330-012-2510-6.

18. Sorace J, Aberle DR, Elimam D, Lawvere S, Tawfik O, Wallace WD. Integrating pathology and radiology disciplines: an emerging opportunity? BMC Med. 2012;10:100. https://doi.org/10.1186/ 1741-7015-10-100.

19. Lippi G, Plebani M. Integrated diagnostics: the future of laboratory medicine? Biochem Med (Zagreb). 2020;30:010501. https://doi. org/10.11613/BM.2020.010501.

Publisher's note Springer Nature remains neutral with regard to jurisdictional claims in published maps and institutional affiliations. 